\title{
Global Estimation of the Cauchy Problem Solutions' the Navier-Stokes Equation
}

\author{
A. A. Durmagambetov, L. S. Fazilova \\ Buketov Karaganda State University, Karaganda, Kazakhstan \\ Email: aset.durmagambet@gmail.com
}

Received 23 January 2014; revised 23 February 2014; accepted 28 February 2014

Copyright (C) 2014 by authors and Scientific Research Publishing Inc.

This work is licensed under the Creative Commons Attribution International License (CC BY). http://creativecommons.org/licenses/by/4.0/

c) (i) Open Access

\begin{abstract}
The analytic properties of the scattering amplitude are discussed, and a representation of the potential is obtained using the scattering amplitude. A uniform time estimation of the Cauchy problem solution for the Navier-Stokes equations is provided.
\end{abstract}

\section{Keywords}

Schrödinger's Equation; Potential; Scattering Amplitude; Cauchy Problem; Navier-Stokes Equations; Fourier Transform

\section{Introduction}

This paper combines the results of studies on the inverse scattering problem with the Cauchy problem for the Navier-Stokes equations. First, we consider some ideas for the potential in the inverse scattering problem, and this is then used to estimate of solutions of the Cauchy problem for the Navier-Stokes equations. A similar approach has been developed for one-dimensional nonlinear equations [1]-[4], but to date, there have been no results for the inverse scattering problem for three-dimensional nonlinear equations. This is primarily due to difficulties in solving the three-dimensional inverse scattering problem.

This paper is organized as follows: first, we study the inverse scattering problem, resulting in a formula for the scattering potential. Furthermore, with the use of this potential, we obtain uniform time estimates in time of solutions of the Navier-Stokes equations, which suggest the global solvability of the Cauchy problem for the Navier-Stokes equations.

Essentially, the present study expands the results for one-dimensional nonlinear equations with inverse scattering methods to multi-dimensional cases. In our opinion, the main achievement is a relatively unchanged projection onto the space of the continuous spectrum for the solution of nonlinear equations, that allows to focus only on the behavior associated with the decomposition of the solutions to the discrete spectrum. In the absence 
of a discrete spectrum, we obtain estimations for the maximum potential in the weaker norms, compared with the norms for Sobolev' spaces.

Consider the operators $H=-\Delta_{x}+q(x), H_{0}=-\Delta_{x}$ defined in the dense set $W_{2}^{2}\left(R^{3}\right)$ in the space $L_{2}\left(R^{3}\right)$, and let $q$ be a bounded fast-decreasing function. The operator $H$ is called Schrödinger's operator.

We consider the three-dimensional inverse scattering problem for Schrödinger's operator: the scattering potential must be reconstructed from the scattering amplitude. This problem has been studied by a number of researchers ([5]-[8] and references therein).

\section{Results}

Consider Schrödinger's equation:

$$
-\Delta_{x} \Psi+q \Psi=|k|^{2} \Psi, k \in C
$$

Let $\Psi_{+}(k, \theta, x)$ be a solution of (1) with the following asympotic behavior:

$$
\Psi_{+}(k, \theta, x)=\mathrm{e}^{\mathrm{i} k \theta x}+\frac{\mathrm{e}^{\mathrm{i}|k| x \mid}}{|x|} A\left(k, \theta^{\prime}, \theta\right)+0\left(\frac{1}{|x|}\right),|x| \rightarrow \infty,
$$

where $A\left(k, \theta^{\prime}, \theta\right)$ is the scattering amplitude and $\theta^{\prime}=\frac{x}{|x|}, \theta \in S^{2}$ for $k \in \bar{C}^{+}=\{\operatorname{Imk} \geq 0\}$

$$
A\left(k, \theta^{\prime}, \theta\right)=-\frac{1}{4 \pi} \int_{R^{3}} q(x) \Psi_{+}(k, \theta, x) \mathrm{e}^{-i k \theta^{\prime} x} \mathrm{~d} x .
$$

Let us also define the solution $\Psi_{-}(k, \theta, x)$ for $k \in \bar{C}^{-}=\{\operatorname{Imk} \leq 0\}$ as

$$
\Psi_{-}(k, \theta, x)=\Psi_{+}(-k,-\theta, x)
$$

As is well known [1]:

$$
\Psi_{+}(k, \theta, x)-\Psi_{-}(k, \theta, x)=-\frac{k}{4 \pi} \int_{S^{2}} A\left(k, \theta^{\prime}, \theta\right) \Psi_{-}\left(k, \theta^{\prime}, x\right) \mathrm{d} \theta^{\prime}, k \in R .
$$

This equation is the key to solving the inverse scattering problem, and was first used by Newton [6] [7] and Somersalo et al. [8].

Equation (4) is equivalent to the following:

$$
\Psi_{+}=S \Psi_{-},
$$

where $S$ is a scattering operator with the kernel $S(k, t), S(k, t)=\int_{R^{3}} \Psi_{+}(k, x) \Psi_{-}^{*}(t, x) \mathrm{d} x$.

The following theorem was stated in [1]:

Theorem 1 (The energy and momentum conservation laws) Let $q \in \mathbf{R}$. Then, $S S^{*}=I, S^{*} S=I$, where I is a unitary operator.

Definition 1 The set of measurable functions $\mathbf{R}$ with the norm, defined by $\|q\|_{\mathbf{R}}=\int_{R^{6}} \frac{q(x) q(y)}{|x-y|^{2}} \mathrm{~d} x \mathrm{~d} y<\infty$ is recognized as being of Rollnik class.

As shown in [8], $\Psi_{ \pm}(k, x)$ is an orthonormal system of $H$ eigenfunctions for the continuous spectrum. In addition to the continuous spectrum there are a finite number $N$ of $H$ negative eigenvalues, designated as $-E_{j}^{2}$ with corresponding normalized eigenfunctions $\psi_{j}\left(x,-E_{j}^{2}\right)(j=\overline{1, N})$, where $\psi_{j}\left(x,-E_{j}^{2}\right) \in L_{2}\left(R^{3}\right)$.

We present Povzner's results [9] below:

Theorem 2 (Completeness) For both an arbitrary $f \in L_{2}\left(R^{3}\right)$ and for $H$ eigenfunctions, Parseval's identity is valid.

$$
|f|_{L_{2}}^{2}=\left(P_{D} f, P_{D} f\right)+\left(P_{A c} f, P_{A c} f\right)
$$




$$
\begin{gathered}
P_{D} f=\sum_{j=1}^{N} f_{j} \psi_{j}\left(x,-E_{j}\right) . \\
P_{A c} f=\int_{0}^{\infty} \int_{S^{2}} s^{2} \bar{f}(s) \Psi_{+}(s, \theta, x) \mathrm{d} \theta \mathrm{d} s,
\end{gathered}
$$

where $\bar{f}$ and $f_{j}$ are Fourier coefficients for the continuous and discrete cases.

Theorem 3 (Birmann-Schwinger estimation). Let $q \in R$. Then, the number of discrete eigenvalues can be estimated as:

$$
N(q) \leq \frac{1}{(4 \pi)^{2}} \int_{R^{3}} \int_{R^{3}} \frac{q(x) q(y)}{|x-y|^{2}} \mathrm{~d} x \mathrm{~d} y .
$$

This theorem was proved in [10].

Let us introduce the following notation:

$$
\begin{gathered}
N A=\int_{S^{2}} A\left(k, \theta^{\prime}, \theta\right) \mathrm{d} \theta, \text { for } f=f\left(k, \theta^{\prime}, x\right), \\
D f=k \int_{S^{2}} A\left(k, \theta^{\prime}, \theta\right) f\left(k, \theta^{\prime}, x\right) \mathrm{d} \theta^{\prime}, \\
\phi_{0}(\sqrt{z}, \theta, x)=\mathrm{e}^{\mathrm{i} \sqrt{2} \theta x}, \\
\Phi\left(\sqrt{z}, \theta^{\prime}, x\right)=\left(\Psi_{+}(\sqrt{z}, \theta, x)-\mathrm{e}^{\mathrm{i} \sqrt{2} \theta x}\right) \Delta,
\end{gathered}
$$

where $\Delta=\prod_{j=1}^{N}\left(k+\mathrm{i} E_{j}\right) /\left(k-\mathrm{i} E_{j}\right)$. We define the operators $T_{ \pm}, T$ for $f \in W_{2}^{1}(R)$ as follows:

$$
\begin{gathered}
T_{+} f=\frac{1}{2 \pi \mathrm{i}} \lim _{\operatorname{Imz} \rightarrow 0} \int_{-\infty}^{\infty} \frac{f(s)}{s-z} \mathrm{~d} s, \operatorname{Im} z>0, \\
T_{-} f=\frac{1}{2 \pi \mathrm{i}} \lim _{\operatorname{Imz} \rightarrow 0} \int_{-\infty}^{\infty} \frac{f(s)}{s-z} \mathrm{~d} s, \operatorname{Im} z<0, \\
T f=\frac{1}{2}\left(T_{+}+T_{-}\right) f .
\end{gathered}
$$

Consider the Riemann problem of finding a function $\Phi$, that is analytic in the complex plane with a cut along the real axis. Values of $\Phi$ on the sides of the cut are denoted as $\Phi_{+}, \Phi_{-}$. The following presents the results of [11]:

Lemma 1

$$
T T=\frac{1}{4} I, T T_{+}=\frac{1}{2} T_{+}, T T_{-}=-\frac{1}{2} T_{-}, T_{+}=T+\frac{1}{2} I, T_{-}=T-\frac{1}{2} I .
$$

Theorem 4 Let $q \in \mathbf{R}, g=\left(\Phi_{+}-\Phi_{-}\right)$. Then,

$$
\Phi_{ \pm}=T_{ \pm} g .
$$

The proof of the above follows from the classic results for the Riemann problem.

Lemma 2 Let $q \in \mathbf{R}, g_{+}=g(\sqrt{z}, \theta, x), g_{-}=g(\sqrt{z},-\theta, x)$. Then,

$$
\Psi_{+}(\sqrt{z}, \theta, x) \Delta=\left(T_{+} g_{+}+\mathrm{e}^{\mathrm{i} \sqrt{2} \theta x}\right), \Psi_{-}(\sqrt{z}, \theta, x) \Delta=\left(T_{-} g_{-}+\mathrm{e}^{-\mathrm{i} \sqrt{z} \theta x}\right) .
$$

The proof of the above follows from the definitions of $g, \Phi_{ \pm}, \Psi_{ \pm}$.

Lemma 3 Let $q \in \mathbf{R}$,

$A_{+}=A(\sqrt{z}, \theta, x), A_{-}=A_{-}(\sqrt{z},-\theta, x)$. Then 


$$
A\left(k, \theta^{\prime}, \theta\right) \Delta=T_{+}\left(A_{+} \Delta-A_{-} \Delta\right) .
$$

The proof of the above again follows from the definitions of the functions $g, \Phi_{ \pm}, \Psi_{ \pm}$.

Lemma 4 Let $q \in \mathbf{R}$. Then,

$$
N A_{+} \Delta=N T_{+}\left(D A \_\Delta\right) .
$$

The proof of the above follows from the definitions of $g, \Phi_{ \pm}, \Psi_{ \pm}$and Theorem 1 .

Definition 2 Denote by $\mathbb{T} \mathbb{A}$ the set of functions $f\left(k, \theta, \theta^{\prime}\right)$ with the norm $\|f\|_{T A}=\sup _{\theta, k, \theta^{\prime}}(|T f|+|f|)<\infty$.

Definition 3 Denote by $\mathbb{R}_{\left(I-D T_{-}\right)}$the set of functions $g$ such that $g=\left(I-D T_{-}\right) f$, for any $f \in \mathbb{T} \mathbb{A}$.

Lemma 5 Suppose $\|A\|_{T A}<\alpha<1$. Then, the operator $\left(I-T_{-} D\right)$, defined on the set $\mathbb{T} \mathbb{A}$ has an inverse defined on $\mathbb{R}_{(I-T-D)}$.

The proof of the above follows from the definitions of $D, T_{-}$and the conditions of Lemma 5 .

Lemma 6 Let $q \in \mathbf{R}$, and assume that $\left(I-T_{-} D\right)^{-1}$ exists. Then,

$$
\begin{gathered}
g=T_{+} g-T_{-} g \\
T_{-} g_{-}=\left(I-T_{-} D\right)^{-1} T_{-} D \phi_{0}, \\
\Psi_{-}=\frac{1}{\Delta}\left(I-T_{-} D\right)^{-1} T_{-} D \phi_{0}+\phi_{0} .
\end{gathered}
$$

The proof of the above follows from the definitions of $g, \Phi_{ \pm}, \Psi_{ \pm}$and Equation (4) Let us rewrite (20) using

$$
\Psi_{ \pm}=\left(K_{ \pm}+I\right) \phi_{0} \text {. }
$$

Lemma 7 Let $q \in \mathbf{R}$. Then,

$$
F_{ \pm}^{-1}=F_{ \pm}^{\prime} \text {, where } F_{ \pm}=K_{ \pm}+I \text {. }
$$

The proof is the same as that in [5].

Lemma 8 Let $q \in \mathbf{R}$. Then,

$$
q=\lim _{z \rightarrow 0} H_{0} \Psi_{-} / \Psi_{-} .
$$

The lemma can be proved by substituting $\Psi_{ \pm}$into Equation (1).

Lemma 9 Let $q \in \mathbf{R}$, and assume that $\left(I-T_{-} D\right)^{-1}$ exists. Then,

$$
q=\lim _{z \rightarrow 0}\left[\frac{1}{\Delta} N\left(I-T_{-} D\right)^{-1} T_{-} D H_{0} \phi_{0}\right] /\left[\frac{1}{\Delta} N\left(I-T_{-} D\right)^{-1} T_{-} D \phi_{0}+N \phi_{0}\right] .
$$

The proof of the above follows from the definitions of $N, \Psi_{ \pm}$and Lemma 6 .

Lemma 10 Let $q \in \mathbf{R}$. Then $\|D\| \leq 2$.

The proof of the above follows from the definition of $D$ and the unitary nature of $S$.

Lemma 11 Let $q \in \mathbf{R} \cap L_{4}\left(R^{3}\right)$. Then,

$$
\begin{gathered}
E_{j}^{2} \leq \int_{R^{3}}\left|q(x) \psi_{j}\right|^{2} \mathrm{~d} x, \\
\max _{x}\left|\psi_{j}(x)\right| \leq 2\left\|q \psi_{j}\right\|_{L_{2}\left(R^{3}\right)^{3}}
\end{gathered}
$$

The proof of the above follows from the definitions of $E_{j}^{2}, \psi_{j}$ and (1).

Lemma 12 Let $q \in \mathbf{R} \cap L_{2}\left(R^{3}\right)$. Then,

$$
\max _{x}\left|P_{D} q\right| \leq 2\|q\|_{L_{2}\left(R^{3}\right)}\|q\|_{\mathbf{R}} \max _{x, j}\left|\psi_{j}(x)\right| .
$$

The proof of the above follows from the definition of $P_{D} f$.

Lemma 13 Let $q \in \mathbf{R} \cap L_{2}\left(R^{3}\right)$, and $\|A\|_{T A}<\alpha<1$. Then, 


$$
\max _{x}\left|P_{A c} q\right| \leq C\|q\|_{L_{2}\left(R^{3}\right)}
$$

To prove this result, one should calculate

$$
\int_{R^{3}} \Psi \Psi \mathrm{d} x=\left(\int_{R^{3}} \Delta \Psi+k^{2} \Psi\right) \mathrm{d} x
$$

Using Lemma 7, the first approximation can be obtained in terms of $\tilde{q}$ :

$$
P_{\text {Ac }} q=\frac{1}{\Delta} T_{-} D \tilde{q}+\mu
$$

where $\mu$ represents terms of highest order of $\tilde{q}$. The lemma can be proved using obvious estimations for $\mu$ and Lemmas 8, 10.

\section{Conclusions for the Three-Dimensional Inverse Scattering Problem}

This study has shown once again the outstanding properties of the scattering operator, which, in combination with the analytical properties of the wave function, allow to obtain an almost-explicit formulas for the potential to be obtained from the scattering amplitude. Furthermore, this approach overcomes the problem of overdetermination, resulting from the fact that the potential is a function of three variables, whereas the amplitude is a function of five variables. We have shown that it is sufficient to average the scattering amplitude to eliminate the two extra variables.

\section{Cauchy Problem for the Navier-Stokes Equation}

Numerous studies of the Navier-Stokes equations are devoted to the problem of the smoothness of its solutions. A good overview of these studies is given in [12]-[14]. The spatial differentiability of the solutions is an important factor, this controls their evolution.

Obviously, differentiable solutions do not provide an effective description of turbulence. On the other hand, the global solvability and differentiability of the solutions has not been proven, and therefore the problem of describing turbulence remains open.

It is interesting to study the properties of the Fourier transform of solutions of the Navier-Stokes equations. Of particular interest is how they can be used in the description of turbulence, and whether they are differentiable. The differentiability of such Fourier transforms appears to be related to the appearance or disappearance of resonance, as this implies the absence of large energy flows from small to large harmonics, which in turn precludes the appearance of turbulence.

Thus, obtaining uniform global estimations of the Fourier transform of solutions of the Navier-Stokes equations means that the principle modeling of complex flows and related calculations will be based on the Fourier transform method.

The authors are continuing to research these issues in relation to a numerical weather prediction model, and this paper is a theoretical justification for this approach.

Consider the Cauchy problem for the Navier-Stokes equations:

$$
\begin{gathered}
q_{t}-v \Delta q+(q, \nabla q)=-\nabla p+f(x, t), \operatorname{div} q=0, \\
\left.q\right|_{t=0}=q_{0}(x)
\end{gathered}
$$

in the domain $Q_{T}=R^{3} \times(0, T)$, where:

$$
\operatorname{div} q_{0}=0 .
$$

The problem defined by (31), (32), (33) has at least one weak solution $(q, p)$ in the so-called Leray-Hopf class [12].

The following results have been proved [12]:

Theorem 5 If

$$
q_{0} \in W_{2}^{1}\left(R^{3}\right), f \in L_{2}\left(Q_{T}\right),
$$

there is a single generalized solution of (31), (32), (33) in the domain $Q_{T_{1}}, T_{1} \in[0, T]$, satisfying the following 
conditions:

$$
q_{t}, \nabla^{2} q, \nabla p \in L_{2}\left(Q_{T}\right)
$$

Note that $T_{1}$ depends on $q_{0}$ and $f$.

Lemma 14 Let $q_{0} \in W_{2}^{1}\left(R^{3}\right), f \in L_{2}\left(Q_{T}\right)$. Then,

$$
\sup _{0 \leq t \leq T}\|q\|_{L_{2}\left(R^{3}\right)}^{2}+\int_{0}^{t}\|\nabla q\|_{L_{2}\left(R^{3}\right)}^{2} \mathrm{~d} \tau \leq\left\|q_{0}\right\|_{L_{2}\left(R^{3}\right)}^{2}+\|f\|_{L_{2}\left(Q_{T}\right)} .
$$

Our goal is to provide global estimations for the Fourier transforms of derivatives of the Navier-Stokes equations' solutions (31), (32), (33) without the that the smallness of the initial velocity and force are small. We obtain the following uniform time estimation. Using the notation that:

$$
\begin{gathered}
\tilde{q}(k)=\int_{R^{3}} q(x) \mathrm{e}^{\mathrm{i}(k, x)} \mathrm{d} x, \tilde{q}(k-l)=\int_{R^{3}} q(x) \mathrm{e}^{\mathrm{i}(k-l, x)} \mathrm{d} x, \\
\tilde{q}_{\text {avg }}(k)=\int_{R^{3}} \tilde{q}(k-l) \delta\left(|k|^{2}-|l|^{2}\right) \mathrm{d} l /|k|,
\end{gathered}
$$

Assertion 1 The solution of (31), (32), (33) according to Theorem 5 satisfies:

$$
\tilde{q}=\tilde{q}_{0}+\int_{0}^{t} \mathrm{e}^{-v|k|^{2}(t-\tau)}([(q, \nabla) q]+\tilde{f}) \mathrm{d} \tau
$$

where $F=-\nabla p+f$.

This follows from the definition of the Fourier transform and the theory of linear differential equations.

Assertion 2 The solution of (31), (32), (33) satisfies:

$$
\tilde{p}=\sum_{i, j} \frac{k_{i} k_{j}}{|k|^{2}} \tilde{q}_{i} q_{j}+i \sum_{i} \frac{k_{i}}{|k|^{2}} \tilde{F}_{i}
$$

and the following estimations:

$$
\begin{gathered}
\|p\|_{L_{2}\left(R^{3}\right)} \leq 3\|\nabla q\|_{L_{2}\left(R^{3}\right)}^{\frac{3}{2}}\|q\|_{L_{2}\left(R^{3}\right)}^{\frac{1}{2}}, \\
|\nabla \tilde{p}| \leq \frac{\left|\tilde{q}^{2}\right|}{|k|}+\frac{|\tilde{f}|}{|k|^{2}}+\frac{1}{|k|}|\nabla \tilde{f}|+3\left|\nabla \tilde{q}^{2}\right| .
\end{gathered}
$$

This expression for $p$ is obtained using div and the Fourier transform. The estimations follow from this representation.

Lemma 15 The solution of (31), (32), (33) in Theorem 5 satisfies the following inequalities:

$$
\begin{aligned}
& \int_{R^{3}}|x|^{2}|q|^{2} \mathrm{~d} x+\int_{0}^{t} \int_{R^{3}}|x|^{2}|\nabla q|^{2} \mathrm{~d} x \mathrm{~d} \tau \leq \text { const, } \\
& \int_{R^{3}}|x|^{4}|q|^{2} \mathrm{~d} x+\int_{0}^{t} \int_{R^{3}}|x|^{4}|\nabla q|^{2} \mathrm{~d} x \mathrm{~d} \tau \leq \text { const, }
\end{aligned}
$$

or

$$
\begin{aligned}
& \|\nabla \tilde{q}\|_{L_{2}\left(R^{3}\right)}+\int_{0}^{t} \int_{R^{3}}|k|^{2}|\tilde{\nabla} q|^{2} \mathrm{~d} k \mathrm{~d} \tau \leq \text { const, } \\
& \left\|\nabla^{2} \tilde{q}\right\|_{L_{2}\left(R^{3}\right)}+\int_{0}^{t} \int_{R^{3}}|k|^{2}\left|\tilde{\nabla}^{2} q\right|^{2} \mathrm{~d} k \mathrm{~d} \tau \leq \text { const. }
\end{aligned}
$$

This follows from the Navier-Stokes equations, our first a priori estimation (Lemma 1) and Lemma 2. 
Lemma 16 The solution of (31), (32), (33) satisfies the following inequalities:

$$
\begin{gathered}
\max _{k}|\tilde{q}| \leq \max _{k}\left|\tilde{q}_{0}\right|+\frac{T}{2} \sup _{0 \leq t \leq T}\|q\|_{L_{2}\left(R^{3}\right)}^{2}+\int_{0}^{t} \|\left.\nabla q\right|_{L_{2}\left(R^{3}\right)} ^{2} \mathrm{~d} \tau, \\
\max _{k}|\nabla \tilde{q}| \leq \max _{k}\left|\nabla \tilde{q}_{0}\right|+\frac{T}{2} \sup _{0 \leq t \leq T}\|\nabla \tilde{q}\|_{L_{2}\left(R^{3}\right)}+\int_{0}^{t} \int_{R^{3}}|k|^{2}|\tilde{\nabla} q|^{2} \mathrm{~d} k \mathrm{~d} \tau, \\
\max _{k}\left|\nabla^{2} \tilde{q}\right| \leq \max _{k}\left|\nabla^{2} \tilde{q}_{0}\right|+\frac{T}{2} \sup _{0 \leq t \leq T}|| \nabla^{2} \tilde{q} \|_{L_{2}\left(R^{3}\right)}+\int_{0}^{t} \int|k|^{2}\left|\nabla^{2} \tilde{q}\right|^{2} \mathrm{~d} k \mathrm{~d} \tau .
\end{gathered}
$$

These estimations follow from (9), Parseval's identity, the Cauchy-Schwarz inequality, and Lemma 3.

Lemma 17 The solution of (31), (32), (33) according to Theorem 5 satisfies $C_{i} \leq$ const, $(i=\overline{0,2,4})$, where:

$$
C_{0}=\int_{0}^{t}\left|\tilde{F}_{1}\right|^{2} \mathrm{~d} \tau, F_{1}=(q, \nabla) q+F, C_{2}=\int_{0}^{t}\left|\nabla \tilde{F}_{1}\right|^{2} \mathrm{~d} \tau, C_{4}=\int_{0}^{t}\left|\nabla^{2} \tilde{F}_{1}\right|^{2} \mathrm{~d} \tau .
$$

This follows from our a priori estimation (Lemma 1) and the assertion of Lemma 3.

Lemma 18 The solution of (31), (32), (33) according to Theorem 5 satisfies to the following inequalities:

$$
\begin{gathered}
\left|\tilde{q}\left(|k|\left(e_{k}-e_{\lambda}\right), t\right)\right| \\
\leq\left|\tilde{q}_{0}\left(|k|\left(e_{k}-e_{\lambda}\right)\right)\right|+\left(\frac{1}{2 v}\right)^{\frac{1}{2}} \frac{C_{0}^{\frac{1}{2}}}{|k|\left|e_{k}-e_{\lambda}\right|},
\end{gathered}
$$

where

$$
C_{0}=\int_{0}^{t}\left|\tilde{F}_{1}\right|^{2} \mathrm{~d} \tau, F_{1}=(q, \nabla) q+F
$$

Proof. From (39), we have the inequality:

$$
\left|\tilde{q}\left(|k|\left(e_{k}-e_{\lambda}\right), t\right)\right| \leq\left|\tilde{q}_{0}\left(|k|\left(e_{k}-e_{\lambda}\right)\right)\right|+\left|\int_{0}^{t} \mathrm{e}^{-v|k|^{2}\left|e_{k}-e_{\lambda}\right|^{2}(t-\tau)} \tilde{F}_{1}\left(|k|\left(e_{k}-e_{\lambda}\right), \tau\right) \mathrm{d} \tau\right|,
$$

where

$$
F_{1}=(q, \nabla) q+F .
$$

Using the notation

$$
I=\left|\int_{0}^{t} \mathrm{e}^{-v|k|^{2}\left|e_{k}-e_{\lambda}\right|^{2}(t-\tau)} \tilde{F}_{1}\left(|k|\left(e_{k}-e_{\lambda}\right), \tau\right) \mathrm{d} \tau\right|,
$$

and Hölder's inequality in $I$, the following inequality can be obtained:

$$
I \leq\left(\int_{0}^{t}\left|\mathrm{e}^{-v|k|^{2}\left|e_{k}-e_{\lambda}\right|^{2}(t-\tau)}\right|^{p} \mathrm{~d} \tau\right)^{\frac{1}{p}}\left(\int_{0}^{t}\left|F_{1}\right|^{q} \mathrm{~d} \tau\right)^{\frac{1}{q}}
$$

where $p, q$ satisfy $\frac{1}{p}+\frac{1}{q}=1$.

Let $p=q=2$. Then,

$$
I \leq\left(\frac{1}{2 v}\right)^{\frac{1}{2}} \frac{\left(\int_{0}^{t}\left|\tilde{F}_{1}\right|^{2} \mathrm{~d} \tau\right)^{\frac{1}{2}}}{|k|\left|e_{k}-e_{\lambda}\right|} .
$$


Using the estimation for $I$ in (57), the assertion in the lemma can be proved.

Lemma 19 Let $q \in R, \max _{k}|\tilde{q}|<\infty$. Then,

$$
\iint_{R^{3} R^{3}} \frac{q(x) q(y)}{|x-y|^{2}} \mathrm{~d} x \mathrm{~d} y \leq C\left(|q|_{L_{2}}+\max _{k}|\tilde{q}|\right)^{2} .
$$

A proof of this lemma can be obtained using Plancherel's theorem.

$$
\begin{aligned}
& \text { For } K=\frac{v^{\frac{1}{2}}}{v^{\frac{1}{2}}-4 \pi C C_{0}^{\frac{1}{2}}} \text { consider the transformation of the Navier-Stokes: } \\
& \qquad t^{\prime}=t A, v^{\prime}=\frac{v}{A}, v^{\prime}=\frac{v}{A}, f^{\prime}=\frac{f}{A^{2}} .
\end{aligned}
$$

Lemma 20 Let $A=\frac{4}{v^{\frac{1}{3}}\left(C C_{0}+1\right)^{\frac{2}{3}}}$, then $K \leq \frac{8}{7}$.

Proof. Using the definitions for $С$ и $C_{0}$ we get

$$
\begin{aligned}
K & =\left(\frac{v}{A}\right)^{\frac{1}{2}}\left(\left(\frac{v}{A}\right)^{\frac{1}{2}}-\frac{4 \pi C C_{0}}{A^{2}}\right)^{-1} \\
& =v^{\frac{1}{2}}\left(v^{\frac{1}{2}}-\frac{4 \pi C C_{0}}{A^{\frac{3}{2}}}\right)^{-1}<\frac{8}{7} .
\end{aligned}
$$

We now obtain uniform time estimations for Rollnik's norms of the solutions of (31), (32), (33). The following (and main) goal is to obtain the same estimations for $\max _{x}|q|$ —velocity components of the Cauchy problem for the Navier-Stokes equations. We will use Lemmas 8 and 13.

Theorem 6 Let $q_{0} \in W_{2}^{2}\left(R^{3}\right), \quad \nabla^{2} \tilde{q}_{0} \in L_{2}\left(R^{3}\right), \quad f \in L_{2}\left(Q_{T}\right), \quad \tilde{f} \in L_{1}\left(Q_{T}\right) \cap L_{2}\left(R^{3}\right)$, $\nabla^{2} \tilde{f} \in L_{1}\left(Q_{T}\right) \cap L_{2}\left(R^{3}\right)$. Then, there exists a unique generalized solution of (31), (32), (33) satisfying the following inequality: $\max _{t} \sum_{i=1}^{3} \max _{x}\left|q_{i}\right| \leq$ const, where the value of const depends only on the conditions of the theorem.

Proof. It suffices to obtain uniform estimates of the maximum velocity components $q_{i}$, which obviously follow from $\max _{x}\left|q_{i}\right|$, because uniform estimates allow us to extend the local existence and uniqueness theorem over the interval in which they are valid. To estimate the velocity components, Lemma 12 can be used:

$$
\begin{gathered}
q_{i}=q_{i} /\left(\int_{0}^{T}\left\|q_{x}\right\|_{L_{2}\left(R^{3}\right)}^{2} \mathrm{~d} t+A_{0}+1\right), \\
A_{0}=4 /\left(v^{\frac{1}{3}}\left(C C_{0}+1\right)^{\frac{2}{3}}\right) .
\end{gathered}
$$

Using Lemmas (15)-(19) for

$$
q_{i}=q_{i} /\left(\int_{0}^{T}\left\|q_{x}\right\|_{L_{2}\left(R^{3}\right)}^{2} \mathrm{~d} t+A_{0}+1\right)
$$

we can obtain $\left\|A_{i}\right\|_{T A}<\alpha<1$, where $A_{i}$ is the amplitude of potential $q_{i}$ and $N\left(q_{i}\right)<1$. That is, discrete solutions are not significant in proving the theorem, so its assertion follows the conditions of Theorem 6 , which defines uniform time estimations for the maximum values of velocity components.

Theorem 6 asserts the global solvability and uniqueness of the Cauchy problem for the Navier-Stokes equations. 


\section{Conclusion}

Uniform global estimations of the Fourier transform of solutions of the Navier-Stokes equations indicate that the principle modeling of complex flows and related calculations can be based on the Fourier transform method. In terms of the Fourier transform, under both smooth initial conditions and right-hand sides, no appear exacerbations appear in the speed and pressure modes. A loss of smoothness in terms of the Fourier transform can only be expected in the case of singular initial conditions, or of unlimited forces in $L_{2}\left(Q_{T}\right)$.

\section{Acknowledgements}

We are grateful to the Ministry of Education and Science of the Republic of Kazakhstan for a grant, and to the System Research "Factor" Company for combining our efforts in this project.

The work was performed as part of an international project, "Joint Kazakh-Indian studies of the influence of anthropogenic factors on atmospheric phenomena on the basis of numerical weather prediction models WRF (Weather Research and Forecasting)”, commissioned by the Ministry of Education and Science of the Republic of Kazakhstan.

\section{References}

[1] Russell, J.S. (1844) Report on Waves. Report of the 14th Meeting of the British Association for the Advancement of Science, York, London, 311.

[2] Russell, J.S. (1838) Report of the Committee on Waves. Report of the 7th Meeting of British Association for the Advancement of Science, John Murray, London, 417-496.

[3] Ablowitz, M.J. (1981) Harvey Segur Solitons and the Inverse Scattering Transform. Studies in Applied and Numerical Mathematics, Philadelphia, 435. http://dx.doi.org/10.1137/1.9781611970883

[4] Zabusky, N.J. and Kruskal, M.D. (1965) Interaction of Solitons in a Collisionless Plasma and the Recurrence of Initial States. Physical Review Letters, 15, 240. http://dx.doi.org/10.1103/PhysRevLett.15.240

[5] Faddeev, L.D. (1974) The Inverse Problem in the Quantum Theory of Scattering. II. Itogi Nauki i Tekhniki. Seriya Sovremennye Problemy Matematiki. Noveishie Dostizheniya, 3, 93-180.

[6] Newton, R.G. (1979) New Result on the Inverse Scattering Problem in Three Dimentions. Physical Review Letters, 43, 541-542. http://dx.doi.org/10.1103/PhysRevLett.43.541

[7] Newton, R.G. (1980) Inverse Scattering Three Dimensions. Journal of Mathematical Physics, 21, 1698-1715. http://dx.doi.org/10.1063/1.524637

[8] Somersalo, E., et al. (1988) Inverse Scattering Problem for the Schrodinger’s Equation in Three Dimensions: Connections between Exact and Approximate Methods.

[9] Povzner, A.Y. (1953) On the Expansion of Arbitrary Functions in Characteristic Functions of the Operator $-\Delta u+c u$. Matematicheskii Sbornik, 74, 109-156.

[10] Birman, M. (1961) On the Spectrum of Singular Boundary-Value Problems. Matematicheskii Sbornik, 55, $125-174$.

[11] Poincare, H. (1910) Lecons de Mecanique Celeste.

[12] Leray, J. (1934) Sur le Mouvement d'un Liquide Visqueux Emplissant l’Espace. Acta Mathematica, 63, 193-248. http://dx.doi.org/10.1007/BF02547354

[13] Ladyzhenskaya, O.A. (1970) Mathematic Problems of Viscous Incondensable Liquid Dynamics. Science, 288.

[14] Solonnikov, V.A. (1964) Estimates Solving Nonstationary Linearized Systems of Navier-Stokes’ Equations. Transactions Academy of Sciences USSR, 70, 213-317. 